\title{
DRIFT CHAMBER CALIBRATION AND TRACK RECONSTRUCTION IN THE P349 ANTIPROTON POLARIZATION EXPERIMENT*
}

D. Alfs, B. GŁowacz, P. Moskal, M. Zieliński

The M. Smoluchowski Institute of Physics, Jagiellonian University Kraków, Poland

D. Grzonka, F. Hauenstein ${ }^{\dagger}$, K. Kilian, D. Lersch

J. Ritman, T. Sefzick

Institut für Kernphysik, Forschungszentrum Jülich, Germany

W. Oelert

Johannes Gutenberg-Universität Mainz, Germany

M. Diermaier, E. Widmann, J. Zmeskal

Stefan-Meyer-Institut für Subatomare Physik, Wien, Austria

M. Wolke

Department of Physics and Astronomy, Uppsala University, Sweden

P. NADEL-TURONSKI

Thomas Jefferson National Accelerator Facility, Newport News, Virginia, USA

M. Carmignotto, T. Horn

Physics Department, The Catholic University of America, Washington DC, USA

H. Mkrtchyan, A. Asaturyan, A. Mkrtchyan, V. Tadevosyan

S. ZHAMKOCHYAN

A.I. Alikhanyan Science Laboratory, Yerevan, Armenia

S. Malbrunot-Ettenauer

CERN, Physics Department, Genve, Switzerland

W. EYRICH

Universität Erlangen, Erlangen, Germany

A. ZINK

Erlangen Centre for Astroparticle Physics (ECAP), Erlangen, Germany

(Received October 3, 2017)

* Presented at the $2^{\text {nd }}$ Jagiellonian Symposium on Fundamental and Applied Subatomic Physics, Kraków, Poland, June 3-11, 2017.

$\dagger$ Present address: Old Dominion University, Norfolk, Virginia, USA. 
The goal of the P349 experiment is to test whether the antiproton production process can be itself a source of antiproton polarization. In this article, we present the motivation and details of the performed measurement. We report on the status of the analysis focusing mainly on calibration of the drift chambers and $3 \mathrm{~d}$ track reconstruction.

DOI:10.5506/APhysPolB.48.1983

\section{Introduction}

Successful experiments with proton beams of energies in the range of a few $\mathrm{GeV}$ have been performed since 1950s while the first polarized proton beam was accelerated at Zero Gradient Synchrotron operated between 1964 and 1979 [1]. For the time being, except for the effortful spin filtering [2, 3], no method for the production of a well-defined polarized antiproton beam with high intensity is available. Some of proposed solutions were already discarded due to expected low-beam intensities or low degree of polarization. In other cases, lack of experimental data (especially the spin-dependent part of the total antiproton-proton scattering cross section) makes it impossible even to estimate the expected efficiency of the proposed method [4].

If antiprotons had a non-zero polarization degree when produced, the preparation of a polarized antiproton beam would be relatively easy. However, until now, there were no dedicated experimental studies of this possibility. The goal of the P349 experiment is to test whether the production process can be itself a source of antiproton polarization $[5,6]$.

\section{Experimental setup}

The experiment was performed at the T11 beamline of the CERN/PS complex. Antiprotons were produced in the reaction $p p \rightarrow \bar{p} p p p$ by bombarding a solid target with an unpolarized proton beam of momentum equal to about $24 \mathrm{GeV} / c$. The experimental setup is presented in the Fig. 1.

\section{Analyzing reaction}

The scattering cross section of a transversely polarized beam is given by

$$
\sigma(\phi)=\sigma_{0}\left(1+A_{y} P \cos \phi\right),
$$

where $\sigma_{0}$ denotes the unpolarized cross section, $P$ stands for polarization and $A_{y}$ is the analyzing power. In the Coulomb Nuclear Interference region, $A_{y}$ is known from experiments and theoretically calculable. The maximum 


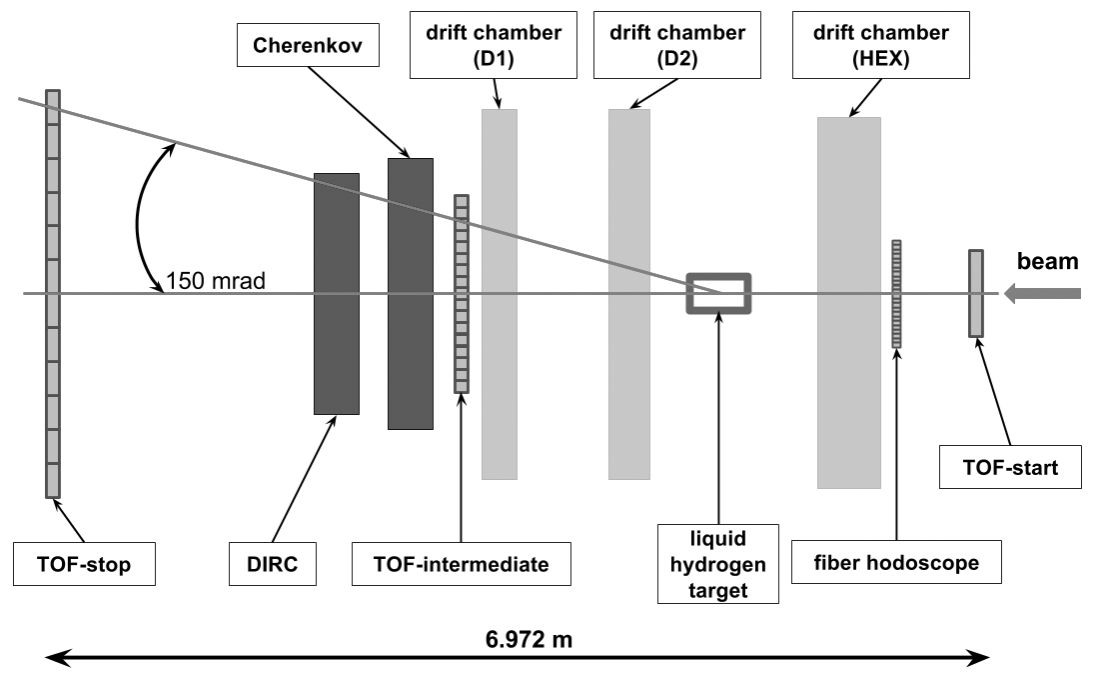

Fig. 1. The scheme of the detector arrangement in the horizontal plane. The beam comes from the right side and it was scattered on a liquid hydrogen target. The setup consisted of scintillators for triggering purposes and time-of-flight measurement, a set of three drift chambers for precise 3d track reconstruction (D1, D2 [7], HEX [8]), a Cherenkov detector for online pion background reduction, a DIRC for offline particle identification and fiber hodoscope for beam monitoring. The total angular range covered by the system is about $150 \mathrm{mrad}$ (horizontally as well as vertically) and the relevant scattering angle for the asymmetry measurement is about 20 mrad.

analyzing power equal to about $4.5 \%$ is reached for a squared four momentum transfer equal to $t=-0.0037(\mathrm{GeV} / c)^{2}$ in high-energy elastic $p p$ collisions [9]. For antiproton scattering, the value is the same but with opposite sign.

In the P349 experiment, the antiprotons momentum spectrum was peaked around $3.5 \mathrm{GeV} / c$. According to preliminary calculations for lower antiproton momenta down to $5 \mathrm{GeV} / c[10]$, the analyzing power for elastic antiproton-proton scattering is expected to be comparable to the highenergy data at laboratory angle between 10 to $20 \mathrm{mrad}$.

\section{Drift chambers calibration}

The calibration is performed iteratively on a sample of events in which all layers of the D1 drift chamber had a signal in exactly one drift cell. First, the drift time spectra offsets are determined and initial drift time - space relations are obtained with the homogeneous irradiation method 
(see left part of Fig. 2). Based on them, the hit positions are calculated and the $3 \mathrm{~d}$ straight tracks for all events are reconstructed. Tracks used in the calibration procedure for a certain layer are obtained without including information from the considered layer (unbiased fit). In order to determine the corrected drift time - space relation the histograms of the residuals, the differences between wire-track and wire-hit distances, are prepared for each layer. In these histograms, for each drift time bin, a projection is made onto the distance axis and a Gaussian function is fitted. For a single time bin, the calibration curve is shifted by the mean value obtained from the fit and one standard deviation of the fitted Gaussian function is considered as the uncertainty of the position determination for the drift times belonging to this bin. With an increasing number of iterations, the values of corrections approach 0 (see right part of Fig. 2).
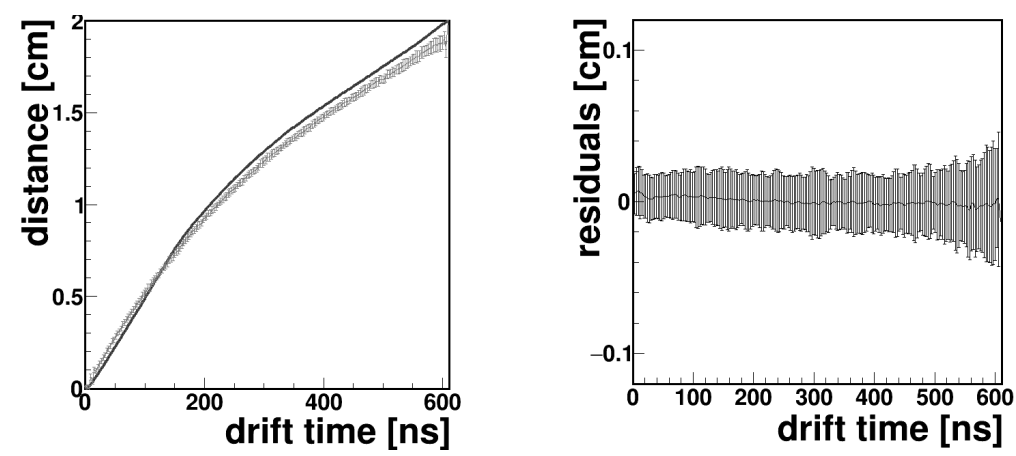

Fig. 2. (Color online) Left: Calibration curve before (darker (blue) line) and after (lighter (red) line) calibration procedure. Right: The mean values of the residuals after 7 iterations. The mean values of the residuals are shown as a thin line around zero together with the uncertainties of their determination. The obtained position uncertainties are in the range of $150-200 \mu \mathrm{m}$.

\section{Track reconstruction}

Track reconstruction relies on the fact that the particle trajectory in the drift chambers is a straight line. Therefore, for its description, the point belonging to the line and a vector of its direction are needed. The parameters are first calculated analytically, then this result is optimized by means of numerical minimization of the expression

$$
\left(\chi^{2}\right)=\sum_{i=1}^{8} \frac{d^{2}\left(r_{x}, r_{y}, a_{x}, a_{y}\right)}{\sigma_{i}^{2}},
$$


where $d$ is the distance between the track and positions of hits calculated within the $i^{\text {th }}$ wire plane with uncertainty equal to $\sigma_{i} ; r_{x, y}$ and $a_{x, y}$ stand for the track point and vector parameters.

The distributions of $3 \mathrm{~d}$ tracks and angles reconstructed from D1 chamber hits are presented in Fig. 3. The two maxima visible in the horizontal track distribution can be identified with two groups of tracks: one going through the analyzing target and another with a direction different from the expected beam intensity maximum. Those tracks originate mainly from scattering at the beamline and its walls [11].
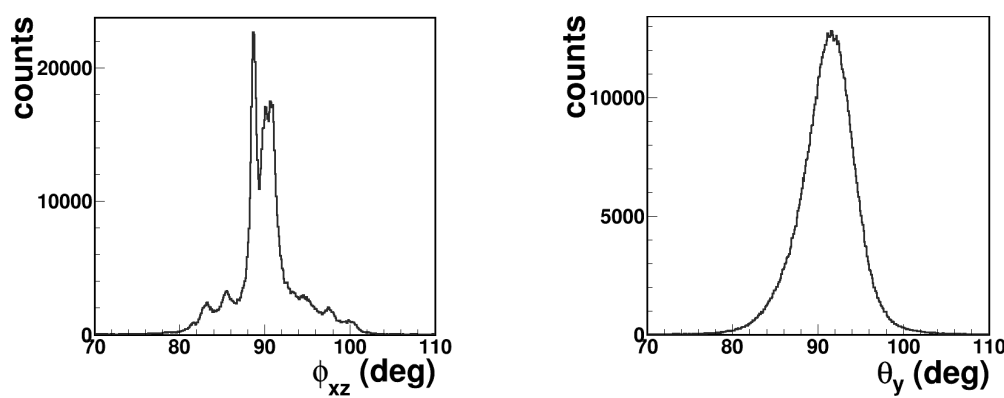

Fig. 3. Angular distribution of tracks in the horizontal (left) and vertical (right) plane. In both cases, $90^{\circ}$ angle corresponds to particles moving perpendicularly to the detector plane.

\section{Summary}

The work on the calibration of the drift chambers and the track reconstruction is ongoing. The main focus now is an analogous D2 and HEX calibration, optimization of the detectors positioning based on reconstruction of unscattered events and selection of events scattered in the angular range of 10 to $20 \mathrm{mrad}$. In the next step, events with antiprotons will be identified and angular distributions of scattered antiproton tracks will be prepared.

This work was supported by the Polish Ministry of Science and Higher Education and by DAAD Exchange Programme from resources of Bundesministerium für Bildung und Forschung (BMBF) through grant PPPPL-57155292, Marian Smoluchowski Kraków Research Consortium "MatterEnergy-Future" (KNOW), and the Polish Ministry of Science and Higher Education through grant 7150/E-338/M/2017. 


\section{REFERENCES}

[1] L.G. Ratner, T.K. Khoe, IEEE Trans. Nucl. Sci. 20, 217 (1973).

[2] P.L. Csonka, Nucl. Instrum. Methods Phys. Res. 63, 247 (1968).

[3] D. Möhl, K. Kilian, CERN/PS/LEA, 1982, p. 701.

[4] E. Steffens, AIP Conf. Proc. 1149, 80 (2009).

[5] K. Kilian et al., Int. J. Mod. Phys. A 26, 757 (2011).

[6] D. Grzonka et al., Acta Phys. Pol. B 46, 191 (2015).

[7] S. Brauksiepe et al., Nucl. Instrum. Methods Phys. Res. A 376, 397 (1996).

[8] J. Smyrski et al., Nucl. Instrum. Methods Phys. Res. A 541, 574 (2005).

[9] H. Okada et al., Phys. Lett. B 638, 450 (2006).

[10] J. Haidenbauer, private communication,

[11] T. Sefzick, private communication. 